\title{
Koolen-De Vries syndrome
}

\author{
INSERM
}

\section{Source}

INSERM. (1999). Orphanet: an online rare disease and orphan drug data base. Koolen-De Vries syndrome. ORPHA:96169

Monosomy 17q21.31 (17q21.31 microdeletion syndrome) is a chromosomal anomaly characterized by developmental delay, childhood hypotonia, facial dysmorphism, and a friendly/amiable behavior. 